\title{
NEUTRON DOSIMETRY (14 MeV) FOR FOIL SAMPLES IRRADIATED WITH THE LA WRENCE LIVERMORE LABORATORY ROTATING TARGET NEUTRON SOURCE
}

\author{
Richard A. Van Konynenburg
}

May 24, 1974

Prepared for U.S. Atomic Energy Commission under contract No.W-7e05-Eng-48

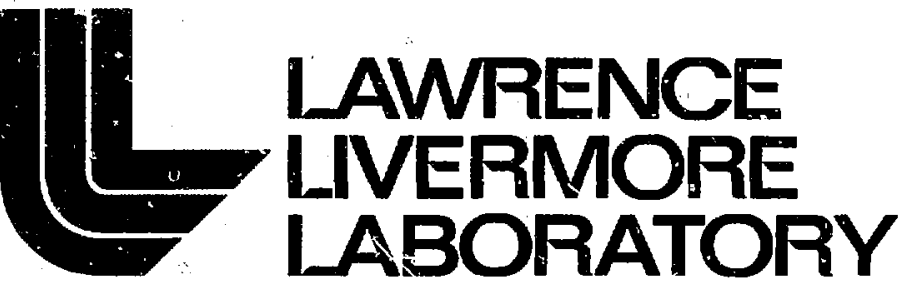

University of Calfornia/Livermore

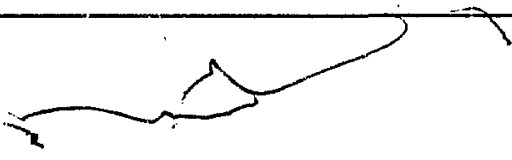




\section{watict:}

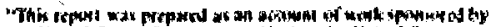

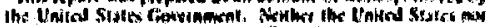

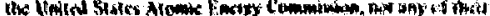

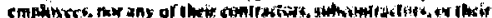

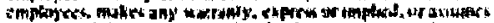

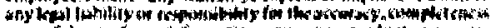

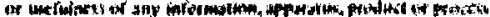

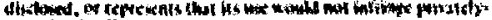
owned tignition

\section{Printed in the United States of America Available from \\ Nitional Technical Information Service \\ U.S. Department of Commerce 5285 Port Royal Road \\ Springfield, Virginia 22151 \\ Price: Printed Copy \$ $\$$; Microfiche \$0.95}

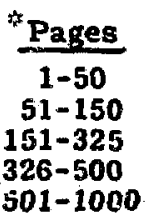

\begin{tabular}{c} 
NTs \\
Selling Priee \\
\hline$\$ 4.00$ \\
$\$ 5.45$ \\
$\$ 7.60$ \\
$\$ 10.60$ \\
$\$ 13.60$
\end{tabular}




\title{
近 \\ LAMAENCE LNERMOAE LABOAATOAY

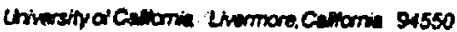

\section{tCRL-51393 Rev. 1 \\ NEUTRON DOSIMETRY (14 MEV) FOR FOIL SAMPLES IRRADIATED WITH THE LAWRENCE LVERAJORE LABORATORY ROTATING TARGET NEUTRON SOURCE}

\author{
Richard A. Van lionynenturg
}

MS. date: May 24, 1974

Supersedes CCRL-51393, dated April 17, 1973

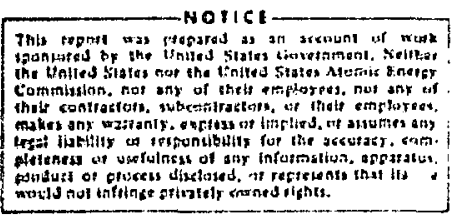




\section{Contents}

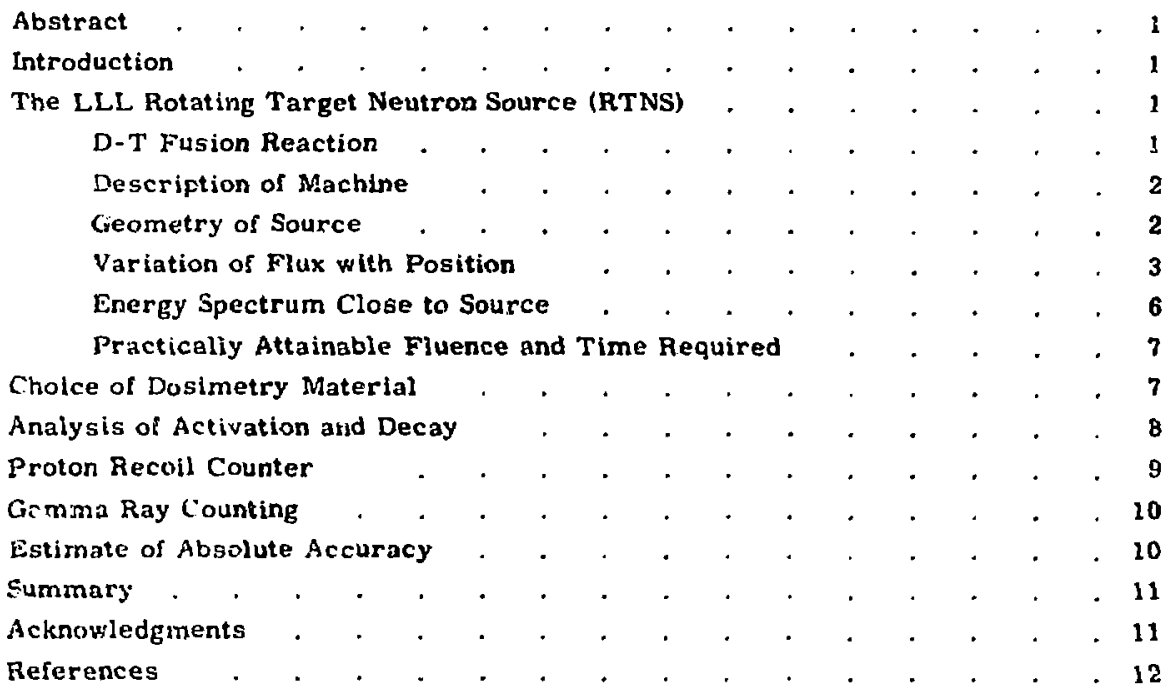




\title{
NEUTRON DOSIMETRY (14 MeV) FOR FOIL SAMPLES IRRADIATED WITH THE LAWRENCE LIVERMORE LABORATORY ROTATING TARGET NEUTRON SOURCE
}

\begin{abstract}
The Lawrence Livermore Laboratory Rotating Target Neutron Source is being used to irradiate small metal and insulator samples to fluences of about $10^{17}$ 14-MeV neutrong $/ \mathrm{cm}^{2}$ as part of radiation-damage studies in support of the Controlled

Thermonuclear Research materials effort. (The quoted value is the nominal energy:

the actual neutron energy depsnds on geometry and ranges up to $15.6 \mathrm{MeV}$.) Because of the characteristics of the neutron souce, foll activation must be used for accurate dosimetry. Niobium is the material of choice for slosimetry. Present techniques yield an absolute accuracy of 7. $5 \%$ in fluence for small samples.
\end{abstract}

\section{Introduction}

To obtain quantitative information about the effects of radiation on materiais, one snust devise methods of accurately determining the dose delivered to samples cluring experiments. Therefore, in support of materials studies for the Controlled Thermonuclear Reactor (CTR) prcjiect at Lawrence Livermore Laboratory, we developed a dosimetry technique for evajuating the flueice of (nominally) 14 - lWIe $V$ neutrons on metal foils and discshaped insulator samples bombarded at the LLL Rotating Target Neutron Source (RTNS). (As discussed below, the actual neutron energy depends on geometry and ranges as high as $15.6 \mathrm{MeV}$.)

First, I will describe the neutron source itself, to provide a background for discussing dosimetry. Following this, 1 will discuss the choice of dosimetry material, analysis of activation and decay, monitoring for dose rate and gamma ray counting. Finally, 1 will estimate the absolute accuracy of this technique.

\section{The LLL Rotating Target Neutron Source (RTNS)}

\section{D-T FUSION REACTION}

The RTNS machine produces neutrons by fusion of deuterium and tritiun ${ }^{l}$ :

${ }_{1}^{2 D}+{ }_{1}^{3} T \rightarrow{ }_{0}^{1} n+\frac{4}{2} \mathrm{He}+17.586 \mathrm{MeV}$
When deuterons are the accelerated species, as is the case in the LLL-RTNS, the cross section for the reaction peaks at a value of atout 5 barns, at a deuteron energy of about $107 \mathrm{keV} .^{2}$ At deuteron energies below $400 \mathrm{keV}$, the reaction is 
isotropic in the center of mass system ${ }^{2}$ and therefore somewhat anisotropic in the laboratory system. In particular, when a $200-\mathrm{keV}$ deuteron interacts with a triton, the relative neutron intensity in the $180^{\circ}$ lab direction is about $86 \%$ of that in the $0^{\circ}$ direction. 2

Although the energy of the neutron is numinally $14 \mathrm{MeV}$ for low deuteron energies, the exact value depends on the deuteron energy and the angle between the path of the incoming deuteron and the path of the emitted neutron. In the RTNS the deuterons are accelerated to an energy of $400 \mathrm{kEV}$ and impinge on a target that is thicker than the deuteron range. Their energy is dissipated primarily by ionization and excitation of electrons. As they are being slowed by these processes, about $0.001 \%$ of the deuterons interact with tritons in the target, producing neutrons. The energy that a deuteron possesses when it undergoes fusion may therefore range from as much as $400 \mathrm{keV}$ to as little as $15 \mathrm{keV}$ or less. The most probable value of this energy depends on the bombarding energy, the energydependence of the $T(d, n){ }^{4} \mathrm{He}$ cross section, the rate of erergy loss in the target, and the tritium loading as a function of target depth. The most probable deuteron energy for a given accelerator can be determined by comparing the neutron energy as a function of angle with theoretically calculated values. This has been done for the LLL-RTNS, and the data are consistent with a most-probable reacting deuteron energy of about $200^{\prime} \mathrm{iV}{ }^{3}$ For a reacting deuteron energy of $200 \mathrm{keV}$, the calculated neutron energy ranges from 13.2 MeV at a laboratory angle of $180^{\circ}$ to 15.1 MeV at an angle of $0^{\circ} .4$

\section{DESCRIPTION OF MACHINE}

The LLL Rotating Target Neutron Scurce has been described in detail elsewhere. ${ }^{5}$ However, the following rough outline is offered as a basis for understanding the need for dosimetry: An Insulated Core Transformer (ICT) accalerator produces a beam of $400-\mathrm{keV}$ deuterons. Up to $17 \mathrm{~mA}$ of this beam passes through a collimator and impinges upon a rotating target composed of 2 thin layer of titanium deposited on a copper backing. The titanium contains tritilim at an average concentration of 1.2 tritium atoms per titanium atom. The target is moursted on a cvlinder that has a rotating seal, ${ }^{6}$ and it revoives at $1100 \mathrm{rpm}$. The target is cooled by water flowing over the copper backing through a thin stainless steel water spreader. The entire target assembly is surrounded by a thin watercatch cage.

\section{GEOMETRY OF SOURCE}

The actual iocalized source of neutrons consists of a disc-shaped region of the target, a few millimeters in diameter and of negligible thickness. The exact size, location, and variation of neutron yield across this apot are somewhat uncertain, and efforts are underway to characterire it more accurately. ${ }^{7,8}$ How ever, an approximate description can be obtained from a consideration of the collimation geometry and previous experiments that included film exposures, ${ }^{7}$ foil activation, ${ }^{9}$ and damage to lucite sample holders. The deuteron collimator in use at the time of writing has a $16-\mathrm{mm}$-square aperture. The deuteron beam itself. is tuned to have 
a $F W H M$ diameter of about $4 \mathrm{~mm}$; its variation in intensity with radius $i 3$ not known exactly, but probably could be approximately described by a Gaussian curve. The beam-deflecting magnets are tuned for maximum neutron count rate on a large plastic scintillation detector located about $1 \mathrm{~m}$ from the target. ${ }^{7}$ During a long irradiation, the beam moves within the aperture and must be periodically retuned. ${ }^{8}$ The effective beam diameter is therefore probably larger than the instantaneous beam diameter but less than the diameter of the collimator aperture.

The distance between the plane of the target spot and the outside of the watercatch cage varies between 2.5 and $4 \mathrm{~mm}$, depending on the alignment of the target and water spreader and on the force pressing the sample hulder against the water-catch cage. A flexible samplemounting assembly has been devised that will conform to irregularities in motion of the target backing while maintaining this distance as small as practicable.

The uniformity of neutron yield across the target spot depends on the variation of current density across the deuteron beam and the variation of tritium loading in the target. Because the target is rotated and scanned radially, the latter factor should not be important, and the time = averaged neuiron yield should correspond only to the beam-current delle ity. Ar pointed out above, the beam also rnoves within its aperture, so that the effect of nonuniformities in beamcurrent density should also be somewhat smoothed out over the course of a long irradiation.
VARIATION OF FLUX WITH TOSITION

Because of the small size of the neutron spot source, the flux decreases rapidly with distance away from it. The variation of flux with position relative to the source can be estimated by representing it as a uniform, isotropically emitting disc of radius $a$ and zero thickness. 9,10 T'he justification for assuming uniformity is given in the "Geometry of Source" section. The assumption of isotropy is valid enough for our calculations for small angular ranges near $0^{\circ}$; for larger angles, this assumption bieaks down, both because of the intrinsic anisotropy (in the laboratory system) of the reaction itselí and because of variation in attenuation by the target backing with change in projected length in the material. This varies with angle and becomes quite significant at $90^{\circ}$. Because of these approximations, and others to be discussed below, the absolute values indicated by these calculations should be regarded as somewhat approximate, but the relative trends should fit the actual situation.

For a point located on the axis of the deuteron beam at a distance $Z$ downstream from the tritiated target, the flux is given by integration over the target spot as

$$
\phi=\frac{Y}{4 \pi a^{2}} \ln \left[1+(\stackrel{a}{i})^{2}\right],
$$

where $\mathrm{Y}$ is the total neutron emission rate over the entire $4 \pi$ solid angle in neutrons/sec. It can be seen that, at large distances, where $Z \gg a$, this expression reduces to $\phi=\frac{Y}{4 \pi Z^{2}}$, as for a point source. 
For a point located off the axis with coordinates $(r, \theta, Z)$ relative to the center of the target spot, the flux is given by

$\phi=\frac{Y}{4 \pi a^{2}} \ln \left[\frac{\sqrt{\left(a^{2}+z^{2}-r^{2}\right)^{2}+4 z^{2} r^{2}}+a^{2}+z^{2}-r^{2}}{2 z^{2}}\right]$.

It can be seen that Eq. (2) reduces to Eq. (1) for $r=0$.

Equation (1) is plotted in Fig. 1 (see Table 1 for detailed diata) for the values $Y=3 \times 10^{12}$ neutrons $/ \mathrm{sec}$ and $a=5 \mathrm{~mm}$. It should be noted that $\mathrm{Y}$ actually decreases with time as i... scribed in Ref. 5. The value chosen here is characteristic of an average well-behaved, fresh target. Also, as noted above, the radius of the spot is not yet well-defined. The value $a=5 \mathrm{~mm}$ is chosen as lying between the instantaneous beam radius and the radius of the collimator aperture.

Tabie 1. Data for Fig. 1.

\begin{tabular}{rc}
\hline $\begin{array}{c}Z, \\
\mathrm{~mm}\end{array}$ & $\begin{array}{c}\text { Flux, } \\
\text { neutrons }\left(\mathrm{cm}^{2}-\mathrm{sec}\right)\end{array}$ \\
\hline 2 & $1.89 \times 10^{12}$ \\
3 & $1.27 \times 10^{12}$ \\
4 & $8.98 \times 10^{11}$ \\
5 & $6.63 \times 10^{11}$ \\
6 & $5.04 \times 10^{11}$ \\
7 & $3.94 \times 10^{11}$ \\
8 & $3.15 \times 10^{11}$ \\
9 & $2.56 \times 10^{11}$ \\
10 & $2.13 \times 10^{11}$ \\
11 & $1.80 \times 10^{11}$ \\
12 & $1.53 \times 10^{11}$ \\
16 & $8.91 \times 10^{10}$ \\
20 & $5.79 \times 10^{10}$ \\
30 & $2.61 \times 10^{10}$ \\
40 & $1.48 \times 10^{10}$ \\
51 & $9.14 \times 10^{9}$ \\
\hline
\end{tabular}

Table 2. Data for Fig. 2.

\begin{tabular}{|c|c|c|c|}
\hline \multirow{2}{*}{$\begin{array}{c}\mathrm{r}, \\
\mathrm{mm}\end{array}$} & \multicolumn{3}{|c|}{$\begin{array}{c}\text { Flux, } \\
\text { neutrons } /\left(\mathrm{cm}^{2}-\mathrm{sec}\right)\end{array}$} \\
\hline & $\mathrm{z}=4 \mathrm{~mm}$ & $\mathrm{z}=16 \mathrm{~mm}$ & $\mathrm{z}=51 \mathrm{~mm}$ \\
\hline 0 & $8.98 \times 10^{11}$ & $8.91 \times 10^{10}$ & $9.14 \times 10^{9}$ \\
\hline 1 & $8.85 \times 10^{11}$ & & \\
\hline 2 & $8.43 \times 10^{11}$ & $8.79 \times 10^{10}$ & $9.12 \times 10^{9}$ \\
\hline 3 & $7.76 \times 20^{11}$ & & \\
\hline 4 & $6.86 \times 10^{11}$ & $8.44 \times 10^{10}$ & $9.09 \times 10^{9}$ \\
\hline 5 & $5.86 \times 10^{11}$ & & \\
\hline 6 & $4.88 \times 10^{11}$ & $7.92 \times 10^{10}$ & $9.02 \times 10^{9}$ \\
\hline 7 & $3.99 \times 10^{11}$ & & \\
\hline 8 & $3.2 \mathrm{~F} \times 10^{11}$ & $7.29 \times 10^{10}$ & $8.92 \times 10^{9}$ \\
\hline 9 & $2.68 \times 10^{11}$ & & \\
\hline 10 & $2.24 \times 10^{11}$ & $6.60 \times 10^{10}$ & $8.80 \times 10^{9}$ \\
\hline 11 & & & \\
\hline 12 & $1.59 \times 10^{11}$ & $5.91 \times 10^{10}$ & $8.67 \times 10^{9}$ \\
\hline 13 & & & \\
\hline 14 & $1.19 \times 10^{11}$ & $5.26 \times 10^{10}$ & $8.50 \times 10^{9}$ \\
\hline
\end{tabular}

Figure 1 shows that the rate of decrease in flux is quite rapid in the range of closest possible approach to the source. This necessitates the use of a thin sample, to obtain a uniform dose. The importance of placing the sample as ? se as possible to the source to maximize the dose is also apparent. Finally, the uncertainty in the source-sample separation distance $\mathrm{Z}$ leads to a large uncertainty in the calculated dose that the sample receives. This has led us to adopt the technique discussed below for measuring the dose directly.

Figure 2 (see Table 2 for detailed data) shows a plot of Eq. (2) for representative values of $Z$ and $r$, using the same values for $\mathrm{Y}$ and $\mathrm{a}$ as in Fig. 1. The successive values of $Z$ are chosen so that the flux for $\mathrm{r}=0$ decreases in increments of about one order of magnitude from one $Z$ value to the next. This plot shows that, using the distance of closest possible approach 


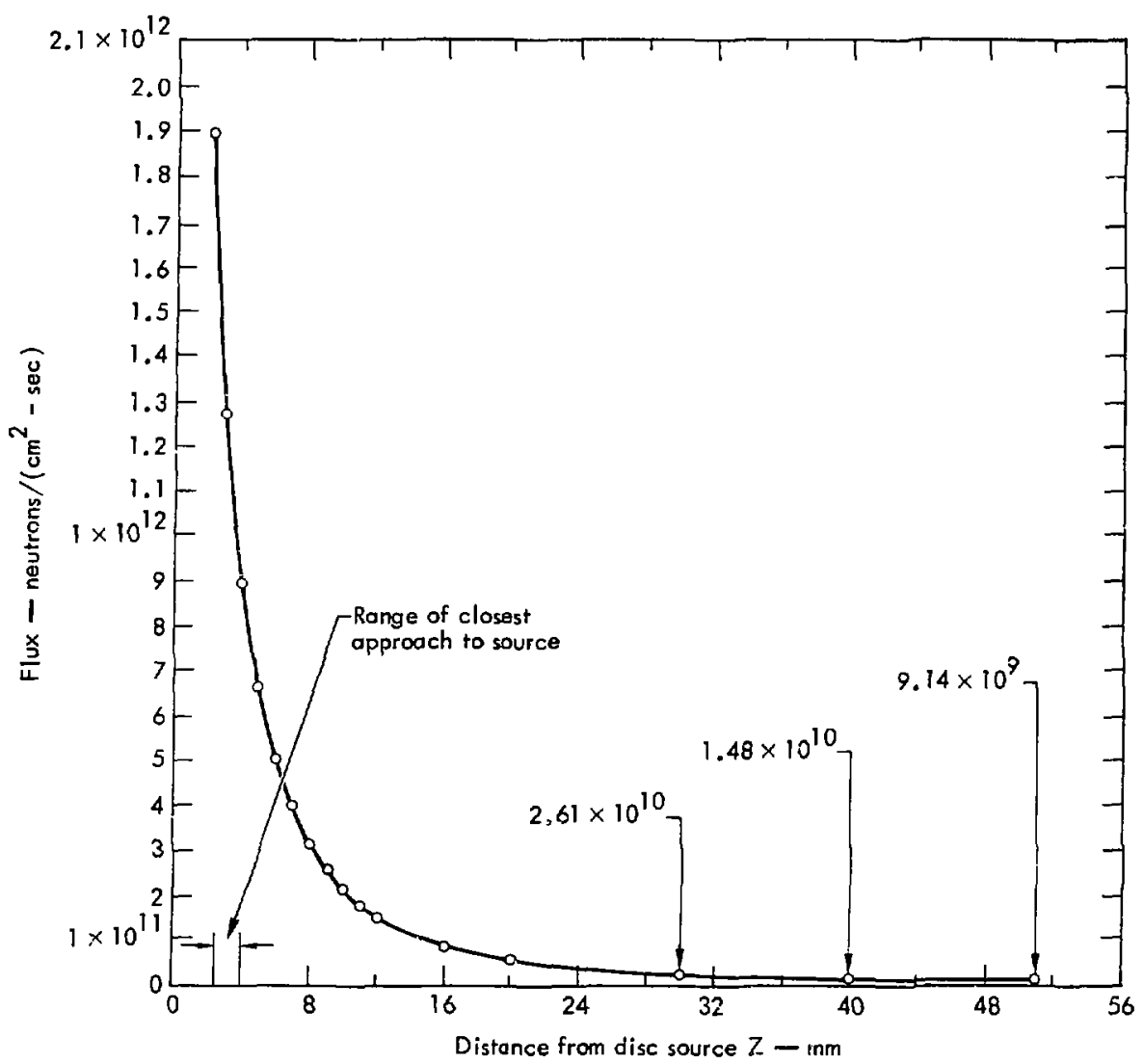

Fig. 1. Calculated variation of flux with distance on-axis $(r=0)$.

(as is customary, to achieve the highest dose), one finds that the lateral uniformity is much decreased, compared to that at greater distances. For example, a $10-\mathrm{mm}-$ diam sample located at $Z=4 \mathrm{~mm}$ receives a dose about $35 \%$ lower at the edge than at the center. This plot also shows that coaxial sample location becomes more critical at close source-sample separations.

In summary, these curves show that, if one desires to achieve the highest pos- sible sample dose, still unif :m across the sample and well-known in absolute value, one must use thin, disc-shaped samples with diameters less than or equal to about $10 \mathrm{~mm}$. They m!'st be placed as closely as possible to the back of the target with accurate axial alignment. Foil activation appears to be the best choice for dosimetry, preferably by using the activation of the sample itself to eliminate the necessity for geometrical and neutronflux attenuation corrections. If this is 


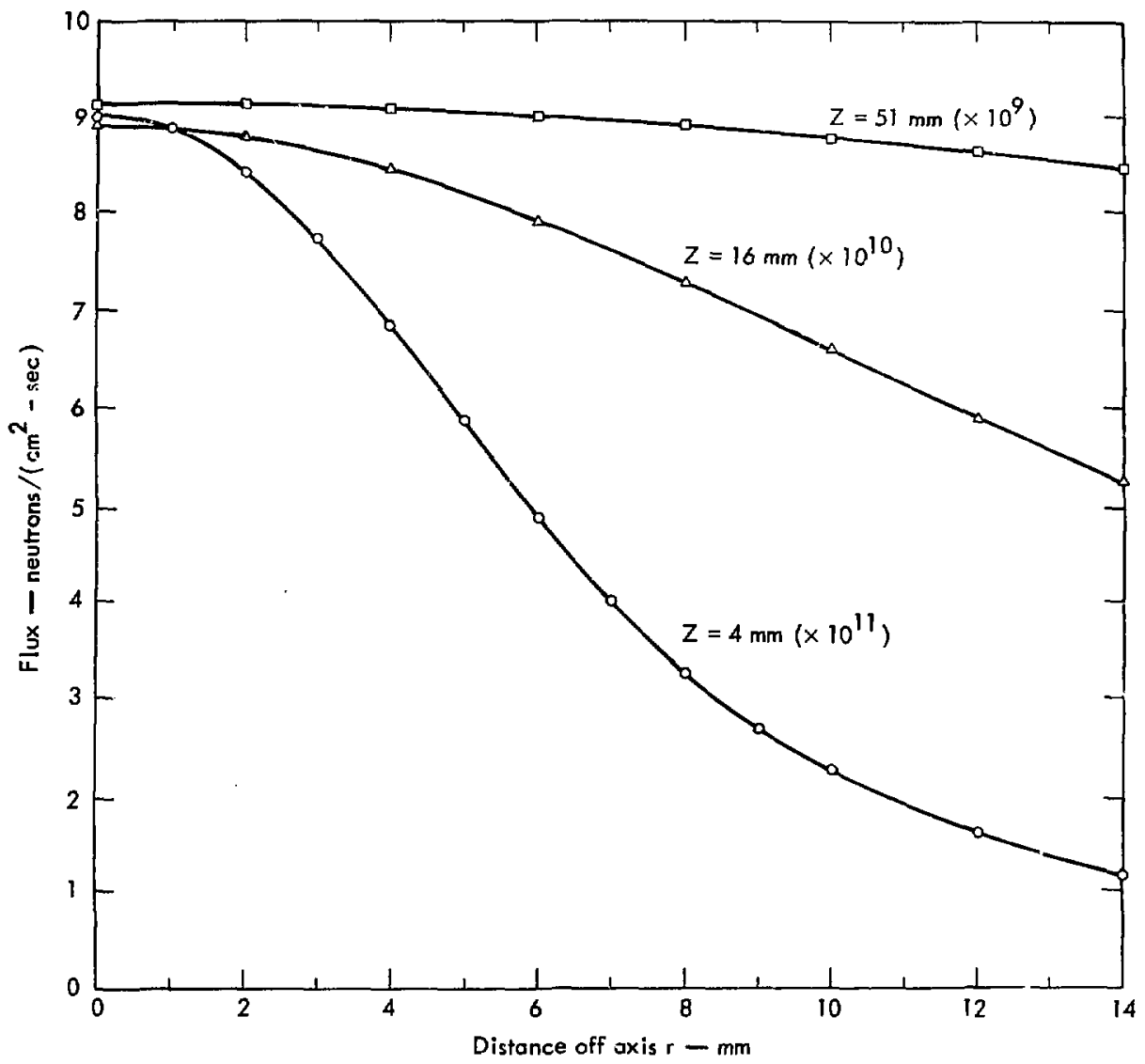

Fig. 2. Calculated variation of flux with distance off axis of source for three values of separation along axis. The multipliers of the values along the ordinate are shown on the curves.

not possible because of unfavorable half life or cross section, a sandwich assembly with thin dosimetry foils in front and in back of the sample appears to be the best apprcach.

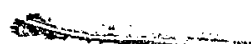
ENERGY SPECTRUM CLOSE TO SOURCE

Because samples are placed on the opposite side of the target from the im- pinging deuteron beam, the maximum neutron energy occurs at an angle of $0^{\circ}$ and is equal to $15.6 \mathrm{MeV}$. However, at distances $Z$ that are small compared to the source diameter $2 \cdot a$, the neutron angle is not sharply defined, so that the ancoubarded by neutrons having a spectrum of energies. In the case of a $10-\mathrm{mm} \cdot$ diam sample placed $4 \mathrm{~mm}$ from the source, the energy ranges from about 
14.1 MeV $t$, $15.6 \mathrm{MeV}$. For this reason, it is desirable that the excitation function of the nucleus used for dosimetry possess little energy-dependence in this range; otherwise, it is necessary to calculate the spectrum from geometrical considerations (which are not well-defined) and integrate over the energy range.

\section{PRACTICALLY ATTAINA BLE} FLUENCE AND TIME REQUIRED

To simulate CTR conditions, it is desirable to attain the highest possible neutron fluence. However, this must be balanced against the cost and time required. For the present accelerator with a 9-in. rotating target, we have somewhat arbitraril $\ddot{y}$ concluded that a maximum practically attainable luence for room temperature irradiations is of the order of $10^{17}$ neutrons $/ \mathrm{cm}^{2}$. This can be achieved on small foil samples in times of less than 1 week, operating with one 8-hr shift per working dey. With around-theclock operation, this fluence could be achieved in less than 2 days. Improvements are being made to increase the average flux, including increases in beam current and iarget diameter, and longer irradiation times could be contemplated if requiled. Linder present conditlons, however, the irradiations last at most a few days.

An activation product used for dosimetry should have a half-life of a few days. If it is much longer, the resulting activity will be reduced. If it is much shorter, scheduling of counting becomes critical. It should be noted that the saturation ef fect is not important in this work, because of the additional information supplied by the proton recoil counter, as discussed below.

\section{Choice of Dosimetry Material}

In 1965 , at a conference held in Texas, "l a standardized dosimetry technique was adopted for use with $14-\mathrm{MeV}$ neutron generators. This technique, 12 known as the "Texas convention," includes the use of copper discs and the reaction ${ }_{29}^{63} \mathrm{Cu}(\mathrm{n}, 2 \mathrm{n}){ }_{29}^{62} \mathrm{Cu}$, which has a cross section of about 500 mbar at $14 \mathrm{MeV}$. The copper discs are bombarded for $1 \mathrm{~min}$. The ${ }_{29}^{62} \mathrm{Cu}$ that is produced decays by positron-emission and has a half-life of $10 \mathrm{~min}$. The $0.511-\mathrm{MeV}$ gamma rays resulting from positron-annihilation are counted and the fluence is calculated, correcting for the duration of irradiation and the time of decay.
This convention is particularly useful for neutron generators in which the flux is low and the irradiation time must be short, but it has the disndvantages of a cross section that is rather encrgydependent ${ }^{13}$ and a short inlf-life that necessitates counting inconveniently soon after the irradiation. Hence, we have chosen to base our dosinetry on the activation of another nuclear species. After considering other candidates, we selected niobium and are using the reaction

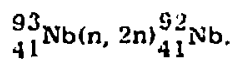

The ${ }_{41}^{92} \mathrm{Nib}$ decays primarily by electron 
capture ${ }^{14}$ to ${ }_{40}^{92} \mathrm{Zr}$, emisting gammil rnys, which are counted after the irradiation.

There are several advantages to this choice:

- The half-life of ${ }_{41}^{92}$ Nib ls 10.10 \pm 0.03 days. ${ }^{t}$ which is in the optimum range for convenience and high induced counting rates.

- The cross section is relatively large and fairly energy-indepentem in the range of interest. Also, it reecently was measurod ${ }^{16}$ to an estimated absolute accuracy of 5 is, based upon the recant

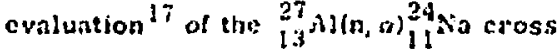
section to an estimated absolute accuracy of better than 1w. The value of the nioblum cross section (for activation of the 10.16-tay isomer only) ranges from $+90=25$ mbil at $14.10 \mathrm{Met}$ to $407 \pm 24$ whar at $14.60 \mathrm{Met}$

- The gamma rays carleded by ${ }^{92} \mathrm{Nb}$ have crorgtes of $0.934,0.912$, and 1.046 Met. making them relietively easy to Actect and cotut.

- Nlobitim occurs naturaliy as a stagle isotupe, ${ }^{14}$ and the cross exctions and half-lives of competing reactions are such that livere are no interferences.

- Noblum is relatively inexpensive (i.e., it is not a precious metal).

- Niobium is a metai of interest to the c"lf materials research program, so that, when ll is the object of study, it can serve as lts own Josimeter.

\section{Analysis of Activation and Decay}

As is well known, the net rate of production of actirated nuclet is given uy

$$
\frac{d N^{\circ}}{d t}=\operatorname{sio}-\lambda N^{\circ} \text {, }
$$

where $\mathrm{N}^{\circ}$ is the number of activated nuclei, $N$ is the number of stable nuelei (assumed constant), is the Rux in nettrons! $\left(\mathrm{cm}^{2}\right.$ sec), $a$ is the cross section in syuare centimeters, and $\lambda$ is the decay constant of the activated spectes in $\mathrm{sec}^{-1}$, which in eurn is equal to 0.093 ' $1 / 2$, where ${ }_{1}, 2$ is the half-lite in scconds.

in our case, o varics as a lunction of time over the duration of the irradiation, because of accelerator shutdowns, tritlum depletion of the target, and variations in accelerstor tuning. However, the use of a proton recoil counter as described in the next section cnables one to obtain a record of the relative neutron output as a function of time. Assuinting that the source-sanple geometry and counter response remain constant, the llux at the sample is given by

$$
\phi(t)=\operatorname{silt}(t) \text {. }
$$

where $R(t)$ is the selescope output and $K$ is a constant to be detcrmined fo: each irratiation, becausc the geometry may vary from one to the next.

It ts desired to obtain the sotal nuence, which is given by

$\Phi=\int_{0}^{t} t(t) d t=k \int_{0}^{t} R(t) d t$. where $i_{f}$ is the ciuration of the irradiation. Equation (3) is tategrated ${ }^{1 B}$ by substltuting Ey. (t) for o and setting $N^{r}$ equal to $X(t) e^{-\lambda t}$, where $X(t)$ is an integrating device. The constant $K$ is then found to be given by 
$K=\frac{N o \int_{0}^{t} \pi(t) e^{x\left(t-t^{\prime}\right)} d t}{\Delta t^{2}\left(t^{t}\right)}$.

The fluence can be expressed ffrom Eqs. (5) and (G)) as

$$
\Phi=\frac{N \sigma \int_{0}^{2} R(t) e^{\pi\left(t-t^{\prime}\right)} d t}{N^{2}(t) d t} .
$$

The data produced by gamma ray counting are in terms of $\mathrm{dN} / \mathrm{dt}$ at the time of counting, $t_{c}$. Since

$$
\left(\frac{\partial N^{0}}{d t}\right)_{t_{c}}=-\lambda N^{0}\left(t_{c}\right)
$$

and

$$
N^{n}\left(t_{c}\right)=N^{n}\left(t_{f}\right) e^{-\lambda\left(t_{c}-t_{f}\right)} .
$$

we lind that

$$
N^{f}\left(t_{c}\right)=-\frac{1}{\lambda}\left(\frac{d N^{0}}{d t}\right)_{t} e^{\lambda\left(t_{c}-t_{t}\right)} .
$$

So that the final expression for the Ruence is

$$
\Phi=-\frac{\left(\frac{\partial N^{0}}{d t}\right)_{t_{c}} e^{\lambda\left(t-t^{-}\right)} \int_{0}^{t_{S}} R(t) d t}{\lambda N \sigma \int_{0}^{t_{f}} R(t) e^{\lambda\left(t-t_{t}\right)} d t} .
$$

The integral in the numerator is equal to the tolal accumulated count from the proton recoll counter. The integral in the denominator is evaluated by summing the contributions of each 1 -hr time segment; within each of them, $R$ is assumed constant. The guantity (d $\mathrm{N}^{\mathrm{b}}$; d $\left.!\right)_{l_{\mathrm{c}}}$ is evaluated by gamma ray counting, as discussed below. The constant $\lambda$ is taken ${ }^{15}$ as $2.843 \times 10^{-3}$ $h r^{-I}$. The number $N$ is evaluated by weighing the foil before irradiation and using the relation

$$
N=\frac{6.0248 \times 10^{23} \mathrm{~m}}{A}
$$

where $m$ is the mass in grams and $A$ is the atomic mass of $\mathrm{Nb}(=92.906)$. The cross section $\sigma$ is taken as $480 \mathrm{mbarn}$; this is obtained by extrapolating the data from Ref. 15 to $15.11 \mathrm{MeV}$ and taking an average value for the cross section between 14.5 and $15.11 \mathrm{MeV}$, weighted toward the higher energies. Although this is admittedly an approximation, it is believed to be valid in view of the foct that the measured cross section. varies only from 498 to 487 mbarn over the energy range from 14.10 to $14.80 \mathrm{MeV}$.

\section{Proton Recoil Counter}

A proton recoil counter is used to provide a continuous count that is directly proportional to the total neutron output (design of such detectors has been described elsewhere ${ }^{19}$ ). The principle of operation is as follows: The 14-Me' neutrons encounter a polyethylene film in the front of the telescope and elastically scatter protons out of it. Some of the protons pass into a scintillation detector. Scattered radiation and background do not present a significart nux in this facility. The peak-to-valley ratio in the recoil 
spectrum is about is to $1 .^{7}$ The efficienc: can be calculated from knowledge of the detector geometry, the composition and thickness of the ilm, and measured values of the differential n-p scattering cross section.

In the present installation, the recoil counter is located at a laboratory angle of about $120^{\circ}$ from the deuteron beam, at a distance of $1.0 \mathrm{~m}$ from the target spot. At this distance, motion of the spot has a negligible cffect on the counting rate. The geometry and efficiency are surh that each count represents a dose of $6.09 \times 10^{7}$ neutrons :

The accunulated count irom the counter is recordiol every liour. Ti,e results are plotted and are used in Eq. (1). Hecause of the information supplied by this counter, saturation with respect to the furmation of $92 \mathrm{Nb}$ does not increase the uncertainty in the calculated fluence, even for irradiation times that are long compared to its half-life, as long as the counting efriciency remains stable. The longtern drift of the counting system efficiency is believed to be less than $5 \%$.

\section{Gamma Ray Counting}

Gamma ray counting is done by personnel of the LLL Radiochemistry Division (see Acknowledgrnents), using a computer-controlled Ge(Li) detector. Corrections are made for self-absorption in the foils, and the geometry is chosen to minimize coincidence losses. Counting periods are normally about $30 \mathrm{~min}$, and time at which the count is performed, $t_{c}$ in Eq. (11), is taken as the mid-time. The absolute accuracy is about $1-2 \%$, including statistical uncertainty and uncertainties due to geometry and detector calibration. $20^{\circ}$

\section{Estimate of Absolute Accuracy}

The two largest possible sources of error in this work are the use of the measured ${ }_{41}^{93} \mathrm{Nb}(\mathrm{n}, 2 \mathrm{n}){ }_{41}^{92} \mathrm{Nb}$ cross section, estimated to be accurate to $5 \%, 16$ and the drift in counting efficiency of the proton recoil counter, estimated at less than $5 \%$. In addition, the gamma ray counting accuracy ${ }^{20}$ is about $1-2 \%$, and errors due to weighing are about $0,5 \%$ for the smallest foils used. Uncertainty in the accuracy of the tabulated half-life of ${ }_{41}^{92} \mathrm{Nb}$ is $0.3 \% .15$
Errors due to measurement of time are considered negligible in comparison. The absolute accuracy of the average sample dose is estimated to be $\pm 7.5 \%$. It should be noted that variation in dose over the lateral dimension of a foll sample can exceed this, as shown in Fig. 2. To be a more accurate measure of dose at various lateral positions, a dosimetry foil can be cut and counted in smaller segments. When a stack of samples is 
used, it is necessary to correct for the shielding effect of the material up-beam from the sample in question.
This is done by means of the cross sections for effective removal of neutrons. 12

\section{Summary}

It has been shown that foil dosimetry is necessary for accurate evaluation of the 14-MeV neutron fluence of samples irradiated close to the target of the LLL Rotating Target Neutron Source. It has also been shown that niobium foils offer particular advantages for the fluences and irradiation times being used in radiation-damage studies for CTR materials research. The resulting procedure yields an absolute accuracy in fluence measurement of $7.5 \%$ for small samples.

\section{Acknowledgments}

I would like to express appreciation to several people who have been and are presently contributing to this effort: Rex Booth-sample mounting assembly, proton recoil counter; David Nethawayadvice in selecting dosimetry foil;

Richard Stuart - help with activation and decay analysis; Ray Gunnink and Ruth Anderson-gamma ray counting; Calvin Wong and E-Division Accelerator StaffRTNS ir radiations: Michael Guinanhelpful discussions; and R.J. Borgidentification of the need for foil dosimetry, and helpful discussions. 


\section{References}

1. A. H. Wapstra, Physica 21, 387 (1955).

2. J. E. Brolley, Jr. and J. L. Fowler, in Fast Neutron Physics, vol. 1, J. B. Marion and J. L. Fowler, Eds. (Interscience, New York, 1960), p. 73.

3. C. Wong, J. D. Anderson, P. Brown, L. F. Hansen, J. L. Kammerdiener, C. Logan, and B. Pohl, Livermore Pulsed Sphere Program: Program Summary Through July 1971, Lawrence Livermore Laboratory, Rept. UCRL-51144 (1971), p. 8 .

4. J. L. Fowler and J. E. Brolley, Jr., Rev. Mod. Phys, 28, 103 (1956).

5. R. Booth and H. H. Barschall, Nucl. Inst. Meth. 99, 1 (1972); and R. Booth, H. H. Barschall, and E. Goldberg, IEEE Trans, Nucl. Sci. NS20, 472 (1973).

6. R. Booth, Nucl. Instr. Meth. 59, 131 (1968).

7. R. Booth, Lawrence Livermore Laboratory, private communication (1974).

8. C. Wong, Lawrence Livermore Laboratory, private communication (1973).

9. E. Goldberg, R. Griffith, and C. Logan, Peak Flux Measurements of the LLL High-Intensity 14-MeV Neutron Source, Lawrence Livermore Laboratory, Rept. UCRL-51317 (1972).

10. J. Op de Beeck, J. Radioanal. Chem. 1, 313 (1968).

11. International Conference on Modern Trends in Activation Analysis, College Station, Texas, Aprll, 1965.

12. D. DeSoete, R. Gijbels, and J. Hoste, Neutron Activation Analysis (WileyInterscience, London, 1972).

13. M. D. Goldberg, S. F. Mughabghab, B. A. Magurno, and V. M. May, Neutron Cross Sections, Vol IIA, $\mathrm{Z}=21$ to 40 , Brookhaven National Laboratory, Rept. BNL 325, 2nd ed., Supp. 2, (1966).

14. C. M. Lederer, J. M. Hollander, and I. Perlman, Table of Isotopes, 6th ed, (John Wiley and Sons, Inc., New York, 1967).

15. M. E. Bunker, B. J. Dropesky, I. D. Knight, and J. W. Starner, Phys. Rev. 127, 844 (1962).

16. D. R. Nethaway, Nucl. Phys. A190, 635 (1972).

17. H. Vonach, M. Hille, G. Steng!, W. Breunlich, and E. Werner, Z. Phys. 237, 155 (1970).

18. R. Stuart, Lawrence Livermore Laboratory, private communication (1972).

19. C. H. Johnson, in Fast Neutron Physics, vol. 1, J. B. Marion and J. L. Fowler, Eds. (Interscience, New York, 1960), p. 247.

20. Ray Gunnink, Lawrence Livermore Laboratory, private communication (1972). 